AIP /123-QED

\title{
An averaging principle for fractional stochastic differential equations with Lévy noise
}

Wenjing $X u, 1, a)$ Jinqiao Duan, ${ }^{2,}$ b) and Wei $X u^{1, c}$ )

1) School of Science, Northwestern Polytechnical University, Xi'an 710129, China

2) Department of Applied Mathematics, Illinois Institute of Technology, Chicago 60616, USA

(Dated: 21 April 2020)

This paper is devoted to the study of an averaging principle for fractional stochastic differential equations in $\mathbb{R}^{n}$ with Lévy motion, using an integral transform method. We obtain a time-averaged equation under suitable assumptions. Furthermore, we show that the solutions of averaged equation approach the solutions of the original equation. Our results in this paper provide better understanding for effective approximation of fractional dynamical systems with non-Gaussian Lévy noise.

Keywords: Stochastic averaging principle, Fractional order systems, Lévy noise

\footnotetext{
a) xuwenjing9121@163.com.

b) duan@iit.edu

c) Author to whom correspondence should be addressed. Electronic mail: weixu@nwpu.edu.cn
} 
Fractional stochastic differential equations are alternative models for anomalous dynamics in various complex systems under non-Gaussian random fluctuations. Although stochastic averaging methods are widely used to gain macroscopic dynamics for multiscale stochastic differential equations, it is still a great challenge to derive effective models to approximate fractional stochastic differential equations, letting alone the systems with non-Gaussian Lévy motion. We take up the challenge to study a fractional averaging principle for a dynamical system with Lévy motion and provide a theoretical foundation of fractional stochastic averaging methods. This offers a reduced yet effective way to accurately predict the solution paths of fractional stochastic systems with Lévy motion, under suitable conditions.

\section{INTRODUCTION}

This paper is devoted to the averaging theory of Caputo type fractional stochastic equations with Lévy motion, looking for an averaged equation in the mean square convergence sense. The theory of averaging are indispensable $\mathrm{e}^{\underline{\underline{3}}}$ that they provide evidences or justifications for the averaging procedures of complex equations arising from mathematics, control, engineering mechanics and several problems. These theoretical results play a crucial role in investigating perturbation theory and nonlinear dynamical systems during their long history.

Stochastic averaging principles, as a kind of effective analysis tool, are presented to help us approach stochastic differential equations (SDEs) with various different noises, such as multi-

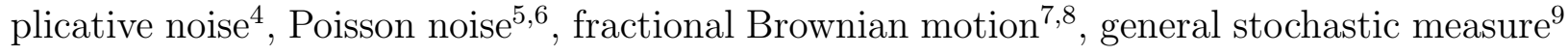
and the like. Lévy noise is an important non-Gaussian noise, and the enthusiasm of researchers are growing in this filed. Recently, $\mathrm{Xu}$ in a literature ${ }^{10}$ gave the integer averaging principle with Lévy noise. However, until now, the fractional averaging behavior with Lévy motion is not well understood.

Fractional derivative, which can characterize the memory and hereditary properties of various practical dynamical systems, has been widespread concerned $\underline{11} \underline{\underline{13}}$. However, most scholars prefer integer order derivative to fractional derivative in the concrete research process, because there is no rigorous mathematic tool. With the in-depth research, they grad- 
ually find that fractional averaging methods can more easily help us simplify and obtain approximate solutions to fractional nonclassical dynamical systems. Roughly speaking, the theory of fractional averaging provides us ample opportunity to accurately reveal the essence of real life $\mathrm{14}$.

Fractional averaging principle is in the innovation phase. In our previous papers 15,16 , by analyses of solutions before and after averaging, we have proved that averaging principles are satisfied for both Caputo fractional stochastic differential equations with Brownian motion and fractional neutral equations with Poisson jumps. Here, we consider the following fractional stochastic equations,

$$
\left\{\begin{aligned}
D_{t}^{\beta} X(t) & =b(t, X(t))+\sigma(t, X(t)) \frac{d L(t)}{d t}, \\
X(0) & =X_{0}
\end{aligned}\right.
$$

where $D_{t}^{\beta}$ is the Caputo fractional derivative, $\beta \in\left(\frac{1}{2}, 1\right)$, initial value $\mathbb{E}\left|X_{0}\right|^{2}<\infty$, functions $b:[0, T] \times \mathbb{R}^{n} \rightarrow \mathbb{R}^{n}$ and $\sigma:[0, T] \times \mathbb{R}^{n} \rightarrow \mathbb{R}^{n \times r}$ are measurable, $L(t)$ is a $r$-dimensional Lévy motion. The above type of equations are of significant importance in applications 17 , which appear in many problems, such as high variability and long memory signal models $\frac{18}{}$, subsurface solute transport ${ }^{19,20}$, infrared remote sensing 21 , among others. They are the ones which attract so much attention since the beginning. We consider in this paper is the averaging character of these substantial interesting equations.

We would like to highlight the fact that our work here is motivated by $\mathrm{Xu}$ et al ${ }^{10}$, who studied the averaging principle with Lévy noise. In this article, we shall generalize the classical Khasminskii averaging approach to Caputo type fractional stochastic equations with Lévy motion.

We first recall some essential definitions and existing results (Section 2). Then we present the fractional averaging results, establishing a stochastic averaging principle for Caputo type fractional equations with Lévy motion (Section 3). Finally, the example is discussed to illustrate the main results (Section 4), and conclusion is given (Section 5).

\section{DEFINITIONS AND EXISTING RESULTS}

Before researching our averaging results, let us give some basic concepts about fractional calculus and Lévy motion. 
Definition II.1. ${ }^{22}$ Let $f$ be a Lebesgue integrable function, $\frac{1}{2}<\beta<1$, the $\beta$-order integral is defined by

$$
I^{\beta} f(t)=\frac{1}{\Gamma(\beta)} \int_{t_{0}}^{t}(t-s)^{\beta-1} f(s) d s, t \in\left[t_{0}, \infty\right),
$$

where $\Gamma$ is the gamma function.

Definition II.2. $\frac{22}{2}$ Let $\frac{1}{2}<\beta<1$, the $\beta$-order Caputo derivative for the function $f$ is

$$
D_{t}^{\beta} f(t)=\frac{1}{\Gamma(1-\beta)} \int_{t_{0}}^{t} \frac{f^{\prime}(s)}{(t-s)^{\beta}} d s, t \in\left[t_{0}, \infty\right) .
$$

Theorem II.1. $\underline{23} \cdot \underline{24}$ If Lévy motion $L(t)$ is in $\mathbb{R}^{r}$, then the expression

$$
L(t)=m t+B(t)+\int_{|x|<c} x \widetilde{N}(t, d x)++\int_{|x| \geq c} x N(t, d x)
$$

is called the Lévy-Itô decomposition, where vector $m \in \mathbb{R}^{r}$, constant $c>0, r$-dimensional Brownian motion $B(t)$ has the covariance matrix $A, N(t, d x): \mathbb{R}^{+} \times\left\{\mathbb{R}^{r}-0\right\}$ controlling small jumps, is Poisson random measure, $\widetilde{N}(t, d x)=N(t, d x)-t \nu(d x)$ controlling large jumps, is compensated Poisson random measure, $\nu$ is the jump measure.

Using the above theorem, let's rewrite equation (11) and give a more general representation

$$
\left\{\begin{aligned}
D_{t}^{\beta} X(t)= & f(t, X(t-))+G(t, X(t-)) \frac{d B(t)}{d t}+\frac{1}{d t} \int_{|x|<c} H(t, X(t-), x) \widetilde{N}(d t, d x) \\
& +\frac{1}{d t} \int_{|x| \geq c} Q(t, X(t-), x) N(d t, d x), \\
X(0)= & X_{0},
\end{aligned}\right.
$$

where functions $f:[0, T] \times \mathbb{R}^{n} \rightarrow \mathbb{R}^{n}, G:[0, T] \times \mathbb{R}^{n} \rightarrow \mathbb{R}^{n \times r}, H:[0, T] \times \mathbb{R}^{n} \times \mathbb{R}^{n} \rightarrow \mathbb{R}^{n}$ and $Q:[0, T] \times \mathbb{R}^{n} \times \mathbb{R}^{n} \rightarrow \mathbb{R}^{n}$ are measurable. According to the technique presented in the literature ${ }^{23}$, we set our sights on the Lévy motion without large jumps,

$$
\left\{\begin{aligned}
D_{t}^{\beta} X(t)= & f(t, X(t-))+G(t, X(t-)) \frac{d B(t)}{d t}+ \\
& \frac{1}{d t} \int_{|x|<c} H(t, X(t-), x) \widetilde{N}(d t, d x) \\
X(0)= & X_{0} .
\end{aligned}\right.
$$

Let us make two assumptions on functions $f, G$ and $H$, 
$\left(H_{1}\right)$ Let $x_{1}, x_{2} \in \mathbb{R}^{n}, t \in[0, T]$ and constant $C_{1}>0$. Then

$$
\begin{aligned}
& \left|f\left(t, x_{1}\right)-f\left(t, x_{2}\right)\right|^{2} \vee\left\|a\left(t, x_{1}, x_{1}\right)-2 a\left(t, x_{1}, x_{2}\right)+a\left(t, x_{2}, x_{2}\right)\right\| \vee \\
& \int_{|x|<c}\left|H\left(t, x_{1}, x\right)-H\left(t, x_{2}, x\right)\right|^{2} \nu(d x) \leq C_{1}\left|x_{1}-x_{2}\right|^{2},
\end{aligned}
$$

where $|\cdot|$ is $\mathbb{R}^{n}$-form, $\|\cdot\|$ is matrix form, $x_{1} \vee x_{2}=\max \left\{x_{1}, x_{2}\right\}$ and $a\left(t, x_{1}, x_{2}\right)=$ $G\left(t, x_{1}\right) G\left(t, x_{2}\right)^{\prime}$ is a $n \times n$ matrix.

$\left(H_{2}\right)$ Let $x_{1} \in \mathbb{R}^{n}, t \in[0, T]$ and constant $C_{2}>0$. Then

$$
\left|f\left(t, x_{1}\right)\right|^{2} \vee\left\|a\left(t, x_{1}, x_{1}\right)\right\| \vee \int_{|x|<c}\left|H\left(t, x_{1}, x\right)\right|^{2} \nu(d x) \leq C_{2}\left(1+\left|x_{1}\right|^{2}\right) .
$$

Conduct assumptions $\left(H_{1}\right)$ and $\left(H_{2}\right)$, equation (2) have the unique, adapted and cadlag mild solution

$$
\begin{aligned}
X(t)= & X_{0}+\frac{1}{\Gamma(\beta)} \int_{0}^{t}(t-s)^{\beta-1} f(s, X(s-)) d s+ \\
& \frac{1}{\Gamma(\beta)} \int_{0}^{t}(t-s)^{\beta-1} G(s, X(s-)) d B(s)+ \\
& \frac{1}{\Gamma(\beta)} \int_{0}^{t}(t-s)^{\beta-1} \int_{|x|<c} H(s, X(s-), x) \widetilde{N}(d s, d x),
\end{aligned}
$$

where $\mathbb{E}\left(\int_{0}^{T}|X(t)|^{2} d t\right)<\infty$. See $\underline{\underline{10} \underline{22}-\underline{25}}$ for more details.

\section{AVERAGING PRINCIPLE FOR FRACTIONAL STOCHASTIC EQUATIONS}

In previous sections, all tools needed for the averaging problem of Caputo type fractional stochastic equations with Lévy noise have been prepared. Start this section by giving the standard form of equation (2):

$$
\left\{\begin{aligned}
D_{t}^{\beta} X_{\epsilon}(t)= & \epsilon f\left(t, X_{\epsilon}(t-)\right)+\sqrt{\epsilon} G\left(t, X_{\epsilon}(t-)\right) \frac{d B(t)}{d t}+ \\
& \frac{\sqrt{\epsilon}}{d t} \int_{|x|<c} H\left(t, X_{\epsilon}(t-), x\right) \tilde{N}(d t, d x), \\
X_{\epsilon}(0)= & X_{0}
\end{aligned}\right.
$$


which is obtained by some time scale transformations. Here, $\epsilon<<1$ is a small positive parameter in $\left(0, \epsilon_{0}\right]$.

Taking the average of functions $f, G, H$ with respect to $t$, we are going to show that the solutions of equation (4) can be approached by the solutions of time-averaged equation

$$
\left\{\begin{aligned}
D_{t}^{\beta} Z_{\epsilon}(t)= & \bar{f}\left(Z_{\epsilon}(t-)\right)+\sqrt{\epsilon} \bar{G}\left(Z_{\epsilon}(t-)\right) \frac{d B(t)}{d t}+ \\
& \frac{\sqrt{\epsilon}}{d t} \int_{|x|<c} \bar{H}\left(Z_{\epsilon}(t-), x\right) \tilde{N}(d t, d x) \\
Z_{\epsilon}(0)= & X_{0}
\end{aligned}\right.
$$

where functions $\bar{f}, \bar{G}, \bar{H}$ satisfying

$\left(H_{3}\right) \quad$ Let $x_{1} \in \mathbb{R}^{n}, T_{1} \in[0, T]$ and $\alpha_{i}\left(T_{1}\right)>0, i=1,2,3$. Then

$$
\begin{gathered}
\left|f\left(T_{1}, x_{1}\right)-\bar{f}\left(x_{1}\right)\right| \leq \alpha_{1}\left(T_{1}\right)\left(1+\left|x_{1}\right|\right), \\
\left\|a\left(T_{1}, x_{1}, x_{1}\right)-\bar{a}\left(x_{1}, x_{1}\right)\right\| \leq \alpha_{2}\left(T_{1}\right)\left(1+\left|x_{1}\right|^{2}\right),
\end{gathered}
$$

and

$$
\int_{|x|<c}\left|H\left(T_{1}, x_{1}, x\right)-H\left(x_{1}, x\right)\right|^{2} \nu(d x) \leq \alpha_{3}\left(T_{1}\right)\left(1+\left|x_{1}\right|^{2}\right),
$$

where $\lim _{T_{1} \rightarrow \infty} \alpha_{i}\left(T_{1}\right)=0$.

Theorem III.1. Let $\delta_{1}>0$ and suppose assumptions $\left(H_{1}\right)$ - $\left(H_{3}\right)$ hold for functions $f, G$ and $H$. Then there exist three constants $L>0, \epsilon_{1} \in\left(0, \epsilon_{0}\right]$ and $\lambda \in(0,1)$ such that

$$
\mathbb{E}\left(\sup _{t \in\left[0, L \epsilon^{-\lambda}\right]}\left|X_{\epsilon}(t)-Z_{\epsilon}(t)\right|^{2}\right) \leq \delta_{1}
$$

for all $\epsilon \in\left(0, \epsilon_{1}\right]$.

Proof. Take a value from $(0, T]$ and define as $u$. Then for any $t \in[0, u]$

$$
\begin{aligned}
& X_{\epsilon}(t)-Z_{\epsilon}(t) \\
= & \frac{\epsilon}{\Gamma(\beta)} \int_{0}^{t}(t-s)^{\beta-1}\left[f\left(s, X_{\epsilon}(s-)\right)-\bar{f}\left(Z_{\epsilon}(s-)\right)\right] d s+ \\
& \frac{\sqrt{\epsilon}}{\Gamma(\beta)} \int_{0}^{t}(t-s)^{\beta-1}\left[G\left(s, X_{\epsilon}(s-)\right)-\bar{G}\left(Z_{\epsilon}(s-)\right)\right] d B(s)+ \\
& \frac{\sqrt{\epsilon}}{\Gamma(\beta)} \int_{0}^{t}(t-s)^{\beta-1} \int_{|x|<c}\left[H\left(s, Z_{\epsilon}(s-), x\right)-\bar{H}\left(Z_{\epsilon}(s-), x\right)\right] \tilde{N}(d s, d x) .
\end{aligned}
$$


Computing the mathematical expectation, we have

$$
\begin{aligned}
& \mathbb{E}\left(\sup _{0 \leq t \leq u}\left|X_{\epsilon}(t)-Z_{\epsilon}(t)\right|^{2}\right) \\
\leq & \frac{3 \epsilon^{2}}{\Gamma(\beta)^{2}} \mathbb{E} \sup _{0 \leq t \leq u}\left|\int_{0}^{t}(t-s)^{\beta-1}\left[f\left(s, X_{\epsilon}(s-)\right)-\bar{f}\left(Z_{\epsilon}(s-)\right)\right] d s\right|^{2}+ \\
& \frac{3 \epsilon}{\Gamma(\beta)^{2}} \mathbb{E} \sup _{0 \leq t \leq u}\left|\int_{0}^{t}(t-s)^{\beta-1}\left[G\left(s, X_{\epsilon}(s-)\right)-\bar{G}\left(Z_{\epsilon}(s-)\right)\right] d B(s)\right|^{2}+ \\
& \frac{3 \epsilon}{\Gamma(\beta)^{2}} \mathbb{E} \sup _{0 \leq t \leq u}\left|\int_{0}^{t}(t-s)^{\beta-1} \int_{|x|<c}\left[H\left(s, X_{\epsilon}(s-), x\right)-\bar{H}\left(Z_{\epsilon}(s-), x\right)\right] \tilde{N}(d s, d x)\right|^{2} \\
= & J_{1}+J_{2}+J_{3} .
\end{aligned}
$$

Evaluating $J_{1}$ with the technic of integration by parts and conditions $\left(H_{1}\right)-\left(H_{3}\right)$, produces

$$
\begin{aligned}
J_{1} \leq & \frac{6 \epsilon^{2}}{\Gamma(\beta)^{2}} \mathbb{E} \sup _{0 \leq t \leq u}\left|\int_{0}^{t}(t-s)^{\beta-1}\left[f\left(s, X_{\epsilon}(s-)\right)-f\left(s, Z_{\epsilon}(s-)\right)\right] d s\right|^{2} \\
& +\frac{6 \epsilon^{2}}{\Gamma(\beta)^{2}} \mathbb{E} \sup _{0 \leq t \leq u}\left|\int_{0}^{t}(t-s)^{\beta-1}\left[f\left(s, Z_{\epsilon}(s-)\right)-\bar{f}\left(Z_{\epsilon}(s-)\right)\right] d s\right|^{2} \\
\leq & \frac{6 \epsilon^{2} u}{\Gamma(\beta)^{2}} \mathbb{E} \sup _{0 \leq t \leq u}\left(\int_{0}^{t}(t-s)^{2 \beta-2}\left|f\left(s, X_{\epsilon}(s-)\right)-f\left(s, Z_{\epsilon}(s-)\right)\right|^{2} d s\right) \\
& +\frac{6 \epsilon^{2}}{\Gamma(\beta)^{2}} \mathbb{E}_{0 \leq t \leq u}\left|\int_{0}^{t}\left[f\left(s, Z_{\epsilon}(s)\right)-\bar{f}\left(Z_{\epsilon}(s)\right)\right] d \frac{-(t-s)^{\beta}}{\beta}\right|^{2} \\
\leq & K_{11} \epsilon^{2} u \int_{0}^{u}(u-s)^{2 \beta-2} \mathbb{E}\left(\sup _{0 \leq s_{1} \leq s}\left|X_{\epsilon}\left(s_{1}\right)-Z_{\epsilon}\left(s_{1}\right)\right|^{2}\right) d s+K_{12} \epsilon^{2} u^{2 \beta},
\end{aligned}
$$

where $K_{11}=\frac{6 C_{1}^{2}}{\Gamma(\beta)^{2}}$ and $K_{12}=\frac{12}{\beta^{2} \Gamma(\beta)^{2}} \sup _{0 \leq t \leq u} \alpha_{1}(t)^{2}\left[1+\mathbb{E}\left(\sup _{0 \leq \tau \leq u}\left|Z_{\epsilon}(\tau)\right|^{2}\right)\right]$.

Similarly, for $J_{2}$,

$$
\begin{aligned}
J_{2} \leq & \frac{6 \epsilon}{\Gamma(\beta)^{2}} \mathbb{E} \sup _{0 \leq t \leq u}\left|\int_{0}^{t}(t-s)^{\beta-1}\left[G\left(s, X_{\epsilon}(s)\right)-G\left(s, Z_{\epsilon}(s)\right)\right] d B(s)\right|^{2}+ \\
& \frac{6 \epsilon}{\Gamma(\beta)^{2}} \mathbb{E} \sup _{0 \leq t \leq u}\left|\int_{0}^{t}(t-s)^{\beta-1}\left[G\left(s, Z_{\epsilon}(s)\right)-\bar{G}\left(Z_{\epsilon}(s)\right)\right] d B(s)\right|^{2},
\end{aligned}
$$

adding Doob's martingale inequality and Itô isometry,

$$
\begin{aligned}
J_{2} \leq & K_{21} \epsilon \int_{0}^{u}(u-s)^{2 \beta-2} \mathbb{E}\left(\sup _{0 \leq s_{1} \leq s}\left|X_{\epsilon}\left(s_{1}\right)-Z_{\epsilon}\left(s_{1}\right)\right|^{2}\right) d s+ \\
& \frac{6 \epsilon}{\Gamma(\beta)^{2}} \mathbb{E} \sup _{0 \leq t \leq u} \int_{0}^{t}\left\|a\left(s, Z_{\epsilon}(s)\right)-\bar{a}\left(Z_{\epsilon}(s)\right)\right\| d\left[\frac{-(t-s)^{2 \beta-1}}{2 \beta-1}\right] \\
\leq & K_{21} \epsilon \int_{0}^{u}(u-s)^{2 \beta-2} \mathbb{E}\left(\sup _{0 \leq s_{1} \leq s}\left|X_{\epsilon}\left(s_{1}\right)-Z_{\epsilon}\left(s_{1}\right)\right|^{2}\right) d s+K_{22} \epsilon u^{2 \beta-1},
\end{aligned}
$$


where $K_{21}=\frac{6 C_{1}^{2}}{\Gamma(\beta)^{2}}$ and $K_{22}=\frac{6}{(2 \beta-1) \Gamma(\beta)^{2}} \sup _{0 \leq t \leq u} \alpha_{2}(t)\left[1+\mathbb{E}\left(\sup _{0 \leq \tau \leq u}\left|Z_{\epsilon}(\tau)\right|^{2}\right)\right]$.

In the sequel, for $J_{3}$,

$$
\begin{aligned}
J_{3} \leq & \frac{6 \epsilon}{\Gamma(\beta)^{2}} \mathbb{E}\left(\int_{0}^{u}(u-s)^{2 \beta-2} \int_{|x|<c}\left|H\left(s, X_{\epsilon}(s-), x\right)-H\left(s, Z_{\epsilon}(s-), x\right)\right|^{2} \nu(d x) d s\right) \\
& +\frac{6 \epsilon}{\Gamma(\beta)^{2}} \mathbb{E}\left(\int_{0}^{u}(u-s)^{2 \beta-2} \int_{|x|<c}\left|H\left(s, X_{\epsilon}(s-), x\right)-\bar{H}\left(Z_{\epsilon}(s-), x\right)\right|^{2} \nu(d x) d s\right),
\end{aligned}
$$

noting the assumptions and formula used above,

$$
\begin{aligned}
J_{3} \leq & K_{31} \epsilon \int_{0}^{u}(u-s)^{2 \beta-2} \mathbb{E}\left(\sup _{0 \leq s_{1} \leq s}\left|X_{\epsilon}\left(s_{1}\right)-Z_{\epsilon}\left(s_{1}\right)\right|^{2}\right) d s+ \\
& \frac{6 \epsilon}{\Gamma(\beta)^{2}} \mathbb{E} \int_{0}^{u} \int_{|x|<c}\left|H\left(s, X_{\epsilon}(s-), x\right)-\bar{H}\left(Z_{\epsilon}(s-), x\right)\right|^{2} \nu(d x) d\left(\frac{-(u-s)^{2 \beta-1}}{2 \beta-1}\right) \\
\leq & K_{31} \epsilon \int_{0}^{u}(u-s)^{2 \beta-2} \mathbb{E}\left(\sup _{0 \leq s_{1} \leq s}\left|X_{\epsilon}\left(s_{1}\right)-Z_{\epsilon}\left(s_{1}\right)\right|^{2}\right) d s+K_{32} \epsilon u^{2 \beta-1},
\end{aligned}
$$

where $K_{31}=\frac{6 C_{1}^{2}}{\Gamma(\beta)^{2}}$ and $K_{32}=\frac{6}{(2 \beta-1) \Gamma(\beta)^{2}} \sup _{0 \leq t \leq u} \alpha_{3}(t)\left[1+\mathbb{E}\left(\sup _{0 \leq \tau \leq u}\left|Z_{\epsilon}(\tau)\right|^{2}\right)\right]$.

Hence, we get

$$
\begin{aligned}
& \mathbb{E}\left(\sup _{0 \leq t \leq u}\left|X_{\epsilon}(t)-Z_{\epsilon}(t)\right|^{2}\right) \\
\leq & K_{12} \epsilon^{2} u^{2 \beta}+\left(K_{22}+K_{32}\right) \epsilon u^{2 \beta-1}+\left(K_{11} \epsilon^{2} u+K_{21} \epsilon+K_{31} \epsilon\right) \\
& \int_{0}^{u}(u-s)^{(2 \beta-1)-1} \mathbb{E}\left(\sup _{0 \leq s_{1} \leq s}\left|X_{\epsilon}\left(s_{1}\right)-Z_{\epsilon}\left(s_{1}\right)\right|^{2}\right) d s,
\end{aligned}
$$

moreover ${ }^{26}, 27$,

$$
\begin{aligned}
& \mathbb{E}\left(\sup _{0 \leq t \leq u}\left|X_{\epsilon}(t)-Z_{\epsilon}(t)\right|^{2}\right) \\
\leq & \left(K_{12} \epsilon^{2} u^{2 \beta}+\left(K_{22}+K_{32}\right) \epsilon u^{2 \beta-1}\right) \\
& \sum_{k=0}^{\infty} \frac{\left[\left(K_{11} \epsilon^{2} u^{1+\beta}+\left(K_{21}+K_{31}\right) \epsilon u^{\beta}\right) \Gamma(\beta)\right]^{k}}{\Gamma(k \beta+1)} .
\end{aligned}
$$

Thus, we can find $L>0$ and $\lambda \in(0,1)$ such that for every $t \in\left(0, L \epsilon^{-\lambda}\right] \subseteq[0, T]$ having

$$
\mathbb{E}\left(\sup _{0 \leq t \leq L \epsilon^{-\lambda}}\left|X_{\epsilon}(t)-Z_{\epsilon}(t)\right|^{2}\right) \leq C \epsilon^{1-\lambda}
$$

where

$$
\begin{aligned}
C= & \left(K_{12} L^{2 \beta} \epsilon^{1+\lambda-2 \beta \lambda}+\left(K_{22}+K_{32}\right) L^{2 \beta-1} \epsilon^{2 \lambda(1-\beta)}\right) \\
& \sum_{k=0}^{\infty} \frac{\left[\left(K_{11} L^{1+\beta} \epsilon^{2-\lambda-\beta \lambda}+\left(K_{21}+K_{31}\right) L^{\beta} \epsilon^{1-\beta \lambda}\right) \Gamma(\beta)\right]^{k}}{\Gamma(k \beta+1)}
\end{aligned}
$$


is a constant.

\section{EXAMPLE}

In this section, we reduce a Caputo type fractional stochastic equations into simpler form to illustrate our main result.

Consider

$$
\left\{\begin{aligned}
D_{t}^{\beta} X_{\epsilon}(t) & =2 \epsilon X_{\epsilon} \cos ^{2}(t)+\sqrt{\epsilon} \frac{d B(t)}{d t}+\sqrt{\epsilon} \int_{|x|<c} 2 x^{4} \sin (t)^{2} X_{\epsilon}(t) \nu_{\alpha}(d x), \\
X_{\epsilon}(0) & =0.1
\end{aligned}\right.
$$

where $\beta \in\left(\frac{1}{2}, 1\right)$, $\alpha$-stable Lévy jump measure $\nu_{\alpha}(d x)=\frac{\gamma}{x^{1+\alpha}} d x$, constants $\gamma>0, \alpha \in(0,2)$.

Together with the averaged system

$$
D_{t}^{\beta} Z_{\epsilon}(t)=\epsilon Z_{\epsilon}\left(1+\gamma_{1}\right)+\sqrt{\epsilon} \frac{d B(t)}{d t}, Z_{\epsilon}(0)=0.1, \gamma_{1}=\frac{\gamma c^{4-\alpha}}{\sqrt{\epsilon}(4-\alpha)}
$$

define

$$
E r=\left[\left|X_{\epsilon}(t)-Z_{\epsilon}(t)\right|^{2}\right]^{\frac{1}{2}}
$$

we numerically compare solution paths for equations (10) and (11) in Fig. 1.

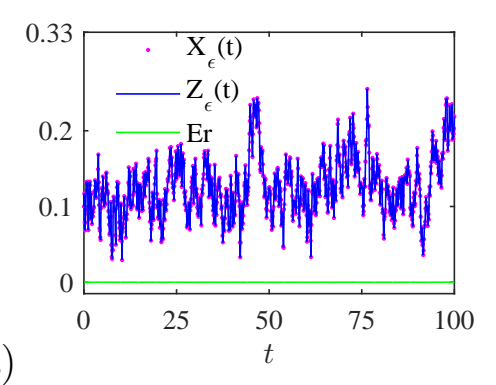

(b)
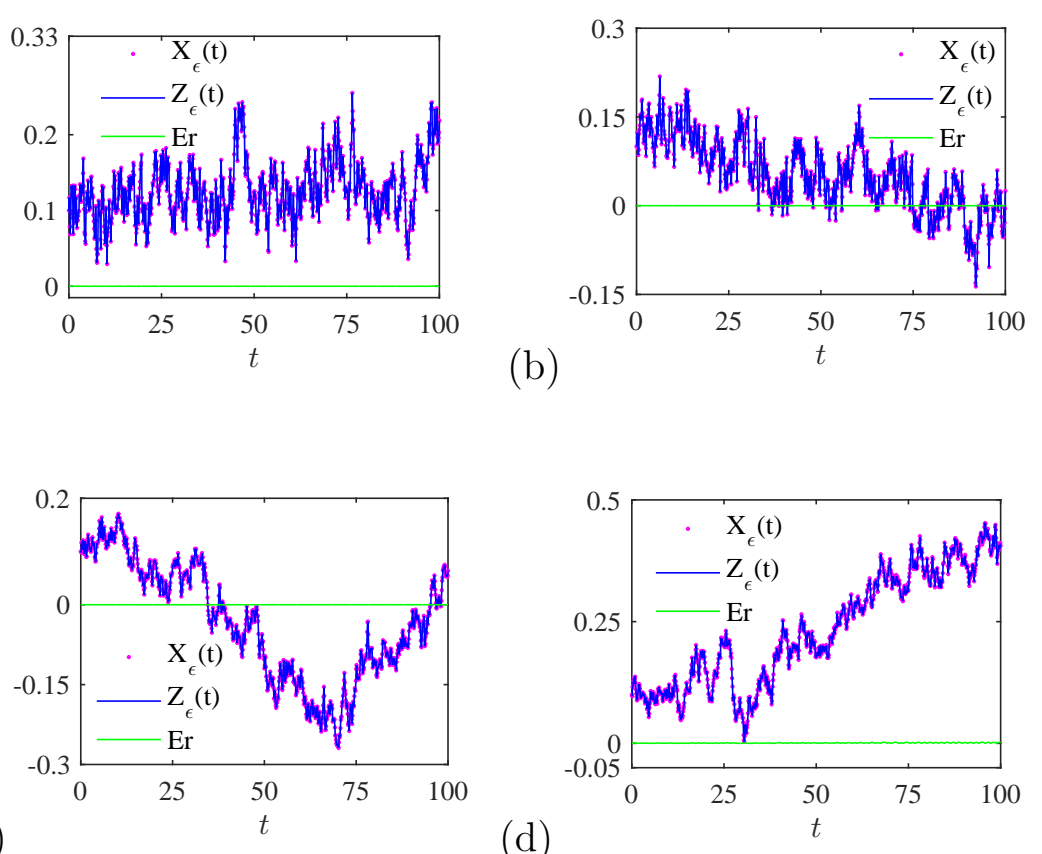

(c)

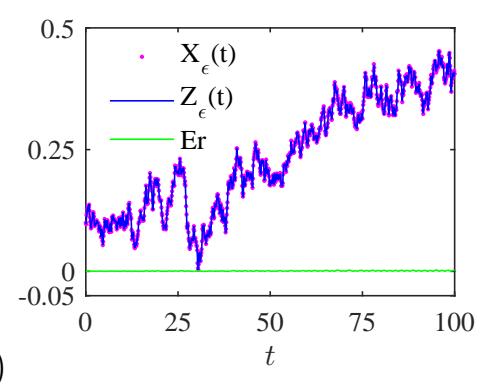


Fig. 1. Solution paths of equations (10) and (11) when $\epsilon=0.001$, $c=0.5:$ (a) $\beta=0.6, \alpha=0.3, \gamma=3$, (b) $\beta=0.6, \alpha=1.1, \gamma=0.6$, (c) $\beta=0.85, \alpha=0.3, \gamma=0.6$, (d) $\beta=0.85, \alpha=1.9, \gamma=3$.

Note that a good agreement is demonstrated.

\section{CONCLUSION}

In this paper, we have proved an averaging principle for the Caputo type fractional stochastic equations with non-Gaussian Lévy motion. It provides an effective stochastic approximation of solutions of fractional stochastic dynamical systems.

As fractional stochastic differential equations arise as models for a variety of complex systems under non-Gaussian random influences, our method for fractional averaging will be beneficial in extracting effective dynamical behaviors of such systems.

\section{ACKNOWLEDGMENTS}

This work was supported by the National Natural Science Foundation of China (No.11872305, 11532011) and China Scholarship Council (No.201906290182).

\section{DATA AVAILABILITY STATEMENT}

The data that support the findings of this study are openly available in GitHub ${ }^{28}$.

\section{REFERENCES}

${ }^{1}$ M. I. Freidlin, A. D. Wentzell. Random perturbations of dynamical systems, 3rd edition, Springer, 2012.

${ }^{2}$ Z. Artstein. Averaging of time-varying differential equations revisited, Journal of Differential Equations, 2007, 243(2): 146-167.

${ }^{3}$ M. Federson, J. G. Mesquita. Non-periodic averaging principles for measure functional differential equations and functional dynamic equations on time scales involving impulses, Journal of Differential Equations, 2013, 255(10): 3098-3126. 
${ }^{4} \mathrm{~S}$. Cerrai. Averaging principle for systems of reaction-diffusion equations with polynomial nonlinearities perturbed by multiplicative noise. SIAM journal on mathematical analysis, 2011, 43(6): 2482-2518.

${ }^{5}$ Y. Zeng, W. Zhu. Stochastic averaging of n-dimensional non-linear dynamical systems subject to non-Gaussian wide-band random excitations, International Journal of NonLinear Mechanics, 2010, 45(5): 572-586.

${ }^{6}$ Y. Zeng, W. Zhu. Stochastic averaging of quasi-linear systems driven by Poisson white noise. Probabilistic engineering mechanics, 2010, 25(1): 99-107.

${ }^{7}$ Y. Xu, B. Pei, J. Wu. Stochastic averaging principle for differential equations with nonLipschitz coefficients driven by fractional Brownian motion. Stochastics and Dynamics, 2017, 17(02): 1750013.

${ }^{8}$ B. Pei, Y. Xu, Y. Bai. Convergence of p-th mean in an averaging principle for stochastic partial differential equations driven by fractional Brownian motion, Discrete \& Continuous Dynamical Systems-Series B, 2020, 25(3).

${ }^{9} \mathrm{~V}$. Radchenko. Strong convergence rate in averaging principle for the heat equation driven by a general stochastic measure, Communications on Stochastic Analysis, 2019, 13(2): 1. ${ }^{10}$ Y. Xu, J. Duan, W. Xu. An averaging principle for stochastic dynamical systems with Lévy noise, Physica D: Nonlinear Phenomena, 2011, 240(17): 1395-1401.

${ }^{11} \mathrm{~V}$. N. Kolokoltsov. Mixed fractional differential equations and generalized operator-valued Mittag-Leffler functions, Mathematical Notes, 2019, 106(5-6): 740-756.

${ }^{12} \mathrm{~V}$. N. Kolokoltsov. The probabilistic point of view on the generalized fractional partial differential equations, Fractional Calculus and Applied Analysis, 2019, 22(3): 543-600.

${ }^{13} \mathrm{~V}$. N. Kolokoltsov. On fully mixed and multidimensional extensions of the Caputo and Riemann-Liouville derivatives, related Markov processes and fractional differential equations, Fractional Calculus and Applied Analysis, 2015, 18(4): 1039-1073.

${ }^{14} \mathrm{~S}$. Chen, J. Shen, L. Wang. Generalized Jacobi functions and their applications to fractional differential equations, Mathematics of Computation, 2016, 85(300): 1603-1638.

${ }^{15} \mathrm{~W}$. Xu, W. Xu, S. Zhang. The averaging principle for stochastic differential equations with Caputo fractional derivative. Applied Mathematics Letters, 2019, 93:79-84.

${ }^{16} \mathrm{~W} . \mathrm{Xu}, \mathrm{W} . \mathrm{Xu}$. An effective averaging theory for fractional neutral stochastic equations of order $0<\alpha<1$ with Poisson jumps. Applied Mathematics Letters, 2020: 106344. 
${ }^{17}$ R. Klages, G. Radons, I. M. Sokolov. Anomalous transport: foundations and applications, John Wiley \& Sons, 2008.

${ }^{18}$ S. M. Kogon, D. G. Manolakis. Signal modeling with self-similar $\alpha$-stable processes: the fractional Lévy stable motion model, IEEE transactions on signal processing, 1996, 44(4): 1006-1010.

${ }^{19}$ S. Lu, F. J. Molz, H. Liu. An efficient, three-dimensional, anisotropic, fractional Brownian motion and truncated fractional Levy motion simulation algorithm based on successive random additions, Computers \& geosciences, 2003, 29(1): 15-25.

${ }^{20}$ H. Liu, G. S. Bodvarsson, S. Lu , et al. A corrected and generalized successive random additions algorithm for simulating fractional Levy motions, Mathematical geology, 2004, 36(3): 361-378.

${ }^{21}$ R. A. Hanel, B. J. Conrath, D. E. Jennings, et al. Exploration of the solar system by infrared remote sensing, Cambridge University Press, 2003.

${ }^{22}$ A. A. Kilbas, H. M. Srivastava, J. J. Trujillo. Theory and Applications of Fractional Differential Equations, Elsevier, 2006.

${ }^{23}$ D. Applebaum. Lévy processes and stochastic calculus, Cambridge University Press, Cambridge, 2009.

${ }^{24}$ J. Duan. An introduction to stochastic dynamics, Cambridge University Press, 2015.

${ }^{25}$ X. Zhang, P. Agarwal, Z. Liu, et al. Existence and uniqueness of solutions for stochastic differential equations of fractional-order $q>1$ with finite delays, Advances in Difference Equations, 2017, 2017(1): 1-18.

${ }^{26} \mathrm{Q}$. Wu. A new type of the Gronwall-Bellman inequality and its application to fractional stochastic differential equations, Cogent Mathematics, 2017, 4(1): 1279781.

${ }^{27}$ H. Ye, J. Gao, Y. Ding. A generalized Gronwall inequality and its application to a fractional differential equation. Journal of Mathematical Analysis \& Applications, 2017, 328(2):10751081.

${ }^{28}$ W. Xu, Code, "https://github.com/wenjinxuxu/averaging', GitHub. 2020. 